\title{
Preliminary Studies towards the Preparation of Reactive 3-Pyrrolin-2-ones in Conjugate Addition Reactions for the Syntheses of Potentially Bioactive 2-Pyrrolidinones and Pyrrolidines
}

\author{
José C. F. Alves* \\ Instituto de Química, Universidade Federal Fluminense, Outeiro de S. João Batista s/n, Centro, \\ 24210-150 Niterói-RJ, Brazil \\ Núcleo de Pesquisas de Produtos Naturais, Universidade Federal do Rio de Janeiro, \\ CCS, Bloco H, 21941-590 Rio de Janeiro-RJ, Brazil
}

\begin{abstract}
Pirrolin-2-onas e 2-pirrolidinonas são subunidades geralmente encontradas na estrutura de vários produtos naturais bioativos e 3-pirrolin-2-onas são valiosos materiais de partida em síntese orgânica devido à habilidade de reagirem como aceptores em reações de adição conjugada. Neste artigo relatamos os resultados iniciais do estudo realizado objetivando as sínteses de 3pirrolin-2-onas reativas em reações de adição conjugada e a preparação de um precursor potencial para a síntese do nootrópico (+/-)-nebracetam.

Pyrrolin-2-ones and 2-pyrrolidinones are moieties often found in the structure of several biologically active natural products and 3-pyrrolin-2-ones are valuable starting materials in organic synthesis due to their ability to react as acceptors in conjugate addition reactions. In this article we report the initial results about the performed study aiming at the syntheses of reactive 3-pyrrolin-2-ones in conjugate addition reactions and the preparation of a potential precursor for the synthesis of the nootropic (+/-)-nebracetam.
\end{abstract}

Keywords: 2-pyrrolidinones, 3-pyrrolin-2-ones, conjugate addition reaction, 2,5dimethoxydihydrofuran, nebracetam

\section{Introduction}

Pyrrolin-2-ones and 2-pyrrolidinones are moieties often found in the structure of several biologically active natural products and 3-pyrrolin-2-ones are valuable starting materials in organic synthesis due to their ability to react as acceptors in conjugate addition reactions of organocuprates, enolates and nitrogen nucleophiles, beyond suffering hydroxylation, epoxidation, cyclopropanation and cycloaddition reactions. ${ }^{1}$ Moreover, 2pyrrolidinones are important key intermediates for the synthesis of pyrrolidines ${ }^{2}$ such as the excitatory amino acids, in which are included kainic acid (KA) and its analogous. ${ }^{3}$ These substances are also important in the field of Medicinal Chemistry, being described in recent literature the syntheses of pyrrolinones and 2-pyrrolidinones as inhibitors of prostate cancer cell growth, ${ }^{4}$ reverse transcriptase ${ }^{5}$ and HIV-1 protease. $^{6}$

*e-mail: alvesjcf@yahoo.com.br
In this article we report a study aiming at the preparation of reactive 3-pyrrolin-2-ones in conjugate addition reactions, for the syntheses of potentially bioactive 2-pyrrolidinones and pyrrolidines.

\section{Results and Discussion}

Initially, we planned to synthesize the $\alpha, \beta$-unsaturated $\gamma$-lactam 4 starting from 2-pyrrolidinone (1) by the two sequences depicted in Scheme 1.

Our initial attempts to get the target molecule 4 by elimination of sulfoxide at compound $\mathbf{3}$ or iodide at the lactam $\mathbf{5}$, followed by protection of nitrogen with the group tertbutoxycarbonyl (Boc), were unsuccessful due to the low yields obtained in the introduction step of the leaving groups at the $\alpha$-position of the respective carbamates. With these unsatisfactory results, we focused our attention to the syntheses of 3-pyrrolin-2-ones with different substitution patterns at the nitrogen atom, using an attractive and convenient procedure in one step from 


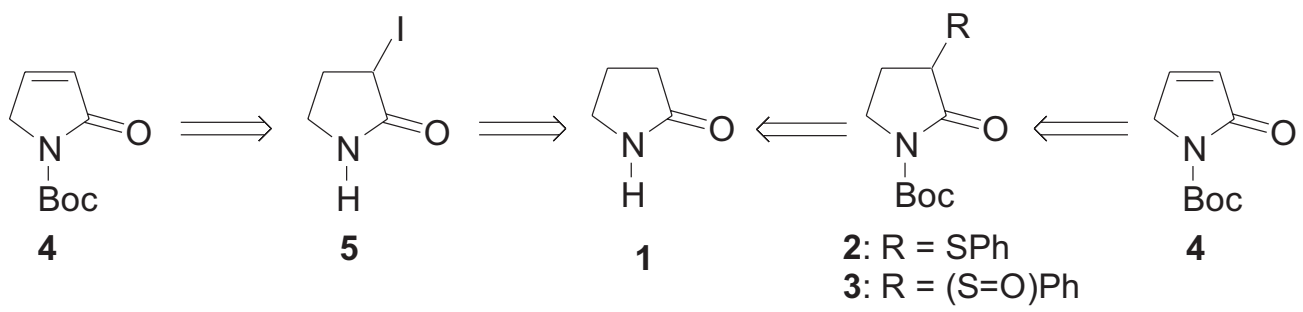

Scheme 1.

2,5-dimethoxydihydrofuran (6) and primary amines (7) (Scheme 2). It was previously proposed that the $\alpha, \beta$-unsaturated $\gamma$-lactams such as $\mathbf{8}$ would be generated from an intramolecular oxido-reduction similar to a Cannizzaro reaction. ${ }^{7}$ Our primary interest was the performance of the condensation of substrate $\mathbf{6}$ with the amines benzylamine (7a), $R$ - $\alpha$-methylbenzylamine (7b) and $S$ - $\alpha$-methylbenzylamine (7c) (Exp. 1-3, Table). After optimization of the reaction conditions, the lactams $8 \mathbf{a}-\mathbf{c}$ were obtained in $55-56 \%$ yield after purification of crude products by column chromatography of silica gel.

Table. Preparation of 3-pyrrolin-2-ones (8a-c)

\begin{tabular}{lcccc}
\hline Exp. & Amine & time/h & Product & $(\%)^{\mathrm{a}}$ \\
\hline 1 & $\mathrm{PhCH}_{2} \mathrm{NH}_{2}$ & 3 & $8 \mathrm{a}$ & 55 \\
2 & & 6 & $8 \mathrm{~b}$ & 56 \\
& & &
\end{tabular}

${ }^{\mathrm{a} A f t e r ~ f l a s h ~ c h r o m a t o g r a p h y ~ o n ~ s i l i c a ~ g e l . ~}$

Initial attempts to add the nucleophiles nitromethane, benzylamine, $R$ - $\alpha$ - and $S$ - $\alpha$-methylbenzylamine to the lactams 8a-c, in a conjugate fashion, were unsuccessful due to the generation of complex mixtures of substances that did not correspond to the expected addition products, as revealed in their NMR spectra. However, previous studies ${ }^{8}$ have demonstrated that the addition of some nucleophilic species to the $\alpha, \beta$-unsaturated system of $\gamma$ lactams requires an electron-withdrawing group attached to the amide nitrogen. Thus, we planned to obtain 3pyrrolin-2-one (9), followed by the introduction of electron-withdrawing Boc as a protective group for the amide nitrogen, aiming at the preparation of $\mathrm{N}$-(tertbutoxycarbonyl)-3-pyrrolin-2-one (4).

The generation of $\gamma$-lactams 8a-c in mild conditions from 2,5-dimethoxydihydrofuran (6), inspired us to investigate this reaction in order to obtain the $\alpha, \beta$ unsaturated $\gamma$-lactam 9 using aqueous ammonium chloride. Indeed, when the condensation reaction of the substrate 6 was performed at conditions depicted in Scheme 3, we obtained a mixture of two substances that were isolated, separated by column chromatography and then identified by spectroscopy methods as the regioisomers 3-pyrrolin2-one (9) and 4-pyrrolin-2-one (10), ${ }^{9}$ with $40 \%$ yield in a respective proportion of $7: 1$.

The transformation of compound 9 into $N$-protected $\gamma$-lactam 4 was carried out in a sequence constituted of deprotonation of the nitrogen with DMAP, followed by reaction of the generated amide ion with (Boc) ${ }_{2} \mathrm{O}$ (Scheme 4 - step $i$ ). ${ }^{10}$ The crude product of this reaction was purified by column chromatography of silica gel to furnish the $\gamma$-lactam 4 in $40 \%$ yield.

With the $\alpha, \beta$-unsaturated $\gamma$-lactam 4 in hand, we passed to the following stage of the reactivity evaluation of this compound as acceptor in conjugate addition reaction. Indeed, the strength of the $\alpha, \beta$-unsaturated system of this compound, with the Boc protection group, was demonstrated by smooth conditions employed at the Nef's reaction to afford the nitro compound 11 (Scheme 4 - step ii). ${ }^{11}$ The $\gamma$-lactam 11 was idealized as a precursor for the synthesis of the nootropic (+/-)-nebracetam (12). ${ }^{12}$

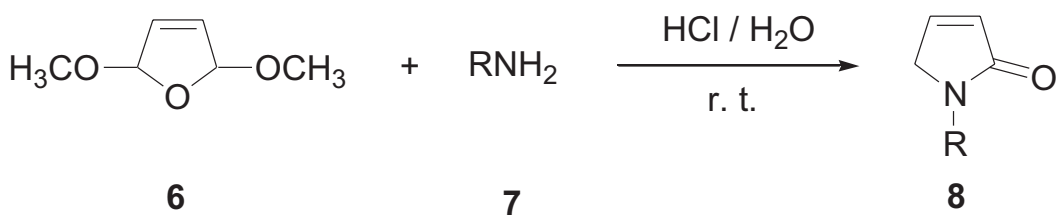
a: $\mathrm{R}=\mathrm{CH}_{2} \mathrm{Ph}$
b: $\mathrm{R}=\mathrm{R}-\alpha-\left(\mathrm{CH}_{3}\right) \mathrm{CHPh}$
c: $\mathrm{R}=\mathrm{S}-\alpha-\left(\mathrm{CH}_{3}\right) \mathrm{CHPh}$

Scheme 2. Reagents: 6 (1.0 equiv.), $\mathrm{RNH}_{2}$ (1.1 equiv.), conc. $\mathrm{HCl}$ ( 0.3 equiv.). 


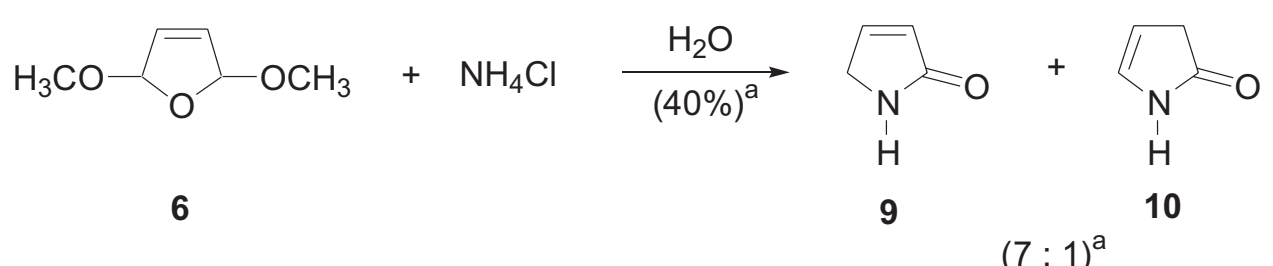

Scheme 3. Reagents and conditions: 6 (1.0 equiv.), $\mathrm{NH}_{4} \mathrm{Cl}$ (5.0 equiv.), $\mathrm{H}_{2} \mathrm{O}$ (r. t. - 1 h); ${ }^{a}$ After flash chromatography on silica gel.

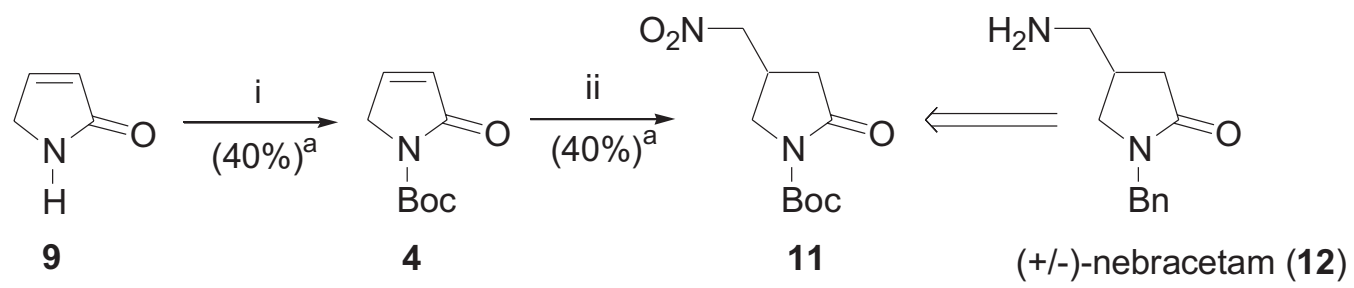

Scheme 4. Reagents and conditions: $i$ ) DMAP (0.05 equiv.), (Boc) $\mathrm{O}_{2}\left(1.1\right.$ equiv.), THF (r. t. - 10 min.); ii) $\mathrm{MeNO}_{2}\left(5.0\right.$ equiv.), DBU (1.0 eq.), $\mathrm{CH}_{3} \mathrm{CN}$ (r. t. - $1 \mathrm{~h}$ ); ${ }^{\mathrm{a} A f t e r}$ flash chromatography on silica gel.

\section{Conclusions}

In summary, we prepared the new 3-pyrrolin-2-ones 8a-c, in one pot procedure, starting from the inexpensive reagent 2,5-dimethoxydihydrofuran (6) and primary amines (7a-c). Although the lactams 8a-c were shown unreactive in conjugate addition reactions, they are ideally adequate as templates for the construction of highly functionalized 2-pyrrolidinones and pyrrolidines, by exploiting functionalization of double bond in some other reactions, such as epoxidation, hydroxylation and cycloadditions besides reduction of the amide group. Using the same methodology for the preparation of compounds 8a-c, we developed a new procedure, in one step, for the synthesis of 3-pyrrolin-2-one (9) from 2,5dimethoxydihydrofuran (6) and aqueous ammonium chloride. The $\alpha, \beta$-unsaturated $\gamma$-lactam 9 was transformed, in two steps, into $\mathrm{N}$-(tert-butoxycarbonyl)-4-nitromethylene-2-pyrrolidinone (11), that can be used as intermediate for further studies towards the synthesis of the nootropic (+/-)-nebracetam (12). Furthermore, the inexpensive one pot procedure used with the substrate $\mathbf{6}$ and aqueous ammonium chloride is appropriate to synthesize in a multigram scale the $\gamma$-lactam $\mathbf{4}$, considered a building block for the syntheses of other potentially bioactive 4-substituted 2-pyrrolidinones as well as the pyrrolidines analogous to kainic acid (KA).

\section{Experimental}

Infrared spectra were recorded on a Nicolet model Magna-IR 760 or Perkin Elmer-1600 model 1605 spectrophotometer (film or $\mathrm{KBr}$ ). ${ }^{1} \mathrm{H}$ NMR and ${ }^{13} \mathrm{C}$ NMR spectra were recorded on a Varian Gemini-200 (200 MHz) or on a Bruker Spectrospin-400 (400 MHz) spectrometer, using $\mathrm{CDCl}_{3}$ or $\mathrm{CD}_{3} \mathrm{OD}$ as the solvent and TMS as internal standard. Coupling constants $(J)$ are reported in Hertz $(\mathrm{Hz})$ and multiplicities are indicated as s (singlet), bs (broad singlet), d (doublet), dd (double doublet), dt (double triplet), q (quartet), m (multiplet). Low Resolution Mass Spectra (LRMS) were obtained by electron-impact (70 $\mathrm{eV}$ ) on a Varian GC-MS Saturn 2000 spectrometer and optical rotations were measured at $25^{\circ} \mathrm{C}$ on a PerkinElmer 243-B polarimeter. Thin layer chromatography was performed on aluminium sheets coated with $60 \mathrm{~F}_{254}$ silica and visualization by UV light and/or for contact of the plates with 7\% ethanolic solution of phosphomolybdic acid and posterior heating. Flash column chromatography was performed on silica gel (230-400 mesh). The solvents and reagents were dried and purified by usual procedures. ${ }^{13}$

General procedure for the preparation of lactams $8 \mathbf{8}-\mathbf{c}$ (Exp. 1-3, Table)

The appropriate amine $7 \mathbf{a} / 7 \mathbf{b} / 7 \mathbf{c}(4.225 \mathrm{mmol})$ was allowed to react with a mixture of 2,5-dimethoxydihydrofuran (6) $(0.500 \mathrm{~g}, 3.841 \mathrm{mmol})$ and conc. $\mathrm{HCl}(0.10$ $\mathrm{mL}, 1.207 \mathrm{mmol})$ as a solution in $\mathrm{H}_{2} \mathrm{O}(3.0 \mathrm{~mL})$ during the respective times indicated at the Table. After the end of reaction, saturated solution of $\mathrm{NaHCO}_{3}$ was added until $\mathrm{pH} 6$ and the mixture was extracted with EtOAc $(3 \times 50$ $\mathrm{mL}$ ). The organic layers were dried with $\mathrm{Na}_{2} \mathrm{SO}_{4}$, filtered, concentrated in vacuum and crude product was then purified by column chromatography eluted with EtOAc.

\section{$N$-Benzyl-3-pyrrolin-2-one (8a) \\ $(0.365 \mathrm{~g}, 55 \%)$ as a brownish oil. $\mathrm{R}_{\mathrm{f}} 0.29$ (EtOAc).} IR (film) $v_{\text {max }} / \mathrm{cm}^{-1}: 3030,2922,1704,1678,1605,1495$, 
$1451,1406,1343,1244,805,700 .{ }^{1} \mathrm{H}$ NMR $(200 \mathrm{MHz}$ $\left.\mathrm{CDCl}_{3}\right): \delta 7.40-7.20(\mathrm{~m}, 5 \mathrm{H}), 7.05(\mathrm{~m}, 1 \mathrm{H}), 6.22(\mathrm{~m}$, $1 \mathrm{H}), 4.64(\mathrm{~s}, 2 \mathrm{H}), 3.87(\mathrm{~m}, 2 \mathrm{H}) .{ }^{13} \mathrm{C} \mathrm{NMR}(50 \mathrm{MHz}$, $\left.\mathrm{CDCl}_{3}\right): \delta 171.08(\mathrm{C}=\mathrm{O}), 142.78(\mathrm{CH}), 136.88(\mathrm{C})$, $128.41(2 \mathrm{CH}), 127.54(2 \mathrm{CH}), 127.43(\mathrm{CH}), 127.24$ $(\mathrm{CH}), 51.98\left(\mathrm{CH}_{2}\right), 45.57\left(\mathrm{CH}_{2}\right) . \mathrm{m} / \mathrm{z}(\%): 173\left(\mathrm{M}^{+}, 97 \%\right)$, 149 (12), 131 (5), 115 (5), 106 (17), 91 (100), 77 (15), 65 (30), 55 (15), 44 (15).

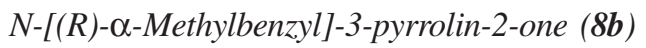

$(0.402 \mathrm{~g}, 56 \%)$ as a brownish oil. $\mathrm{R}_{\mathrm{f}} 0.37$ (EtOAc). $[\alpha]_{\mathrm{D}}^{25}+114\left(\mathrm{c} 1.20, \mathrm{CH}_{3} \mathrm{OH}\right)$. IR (film) $v_{\max } / \mathrm{cm}^{-1}: 3030$, 2977, 1687, 1666, 1586, 1495, 1447, 1400, 1240, 802, 701. ${ }^{1} \mathrm{H}$ NMR (200 MHz, $\left.\mathrm{CDCl}_{3}\right): \delta 7.40-7.21(\mathrm{~m}, 5 \mathrm{H})$, $7.03(\mathrm{~m}, 1 \mathrm{H}), 6.18(\mathrm{~m}, 1 \mathrm{H}), 5.58(\mathrm{q}, J 7.1 \mathrm{~Hz}, 1 \mathrm{H})$, $3.94(\mathrm{dt}, J 1.8$ and $20.4 \mathrm{~Hz}, 1 \mathrm{H}), 3.62(\mathrm{dt}, J 1.8$ and $20.4 \mathrm{~Hz}, 1 \mathrm{H}), 1.61(\mathrm{~d}, J 7.1 \mathrm{~Hz}, 3 \mathrm{H}) .{ }^{13} \mathrm{C}$ NMR $(100$ $\left.\mathrm{MHz}, \mathrm{CDCl}_{3}\right): \delta 170.76(\mathrm{C}=\mathrm{O}), 142.65(\mathrm{CH}), 140.66$ $(\mathrm{C}), 128.29(2 \mathrm{CH}), 127.45(\mathrm{CH}), 127.14(\mathrm{CH}), 126.54$ $(2 \mathrm{CH}), 48.72(\mathrm{CH}), 48.41\left(\mathrm{CH}_{2}\right), 17.43\left(\mathrm{CH}_{3}\right) . \mathrm{m} / \mathrm{z}(\%)$ : $187\left(\mathrm{M}^{+}, 80 \%\right), 172$ (100), $160(40), 149(15), 132(10)$, 117 (15), 105 (97), 96 (17), 77 (60), 69 (17), 51 (35), 44 (17).

\section{$N-[(S)$ - $\alpha$-Methylbenzyl]-3-pyrrolin-2-one $(8 c)$}

$(0.402 \mathrm{~g}, 56 \%)$ as a brownish oil. $\mathrm{R}_{\mathrm{f}} 0.37$ (EtOAc). $[\alpha]_{\mathrm{D}}^{2.5}$ -107 (c 1.12, $\left.\mathrm{CH}_{3} \mathrm{OH}\right)$.

\section{3-Pyrrolin-2-one (9) and 4-pyrrolin-2-one (10)}

To a solution of $\mathrm{NH}_{4} \mathrm{Cl}(6.162 \mathrm{~g}, 115.225 \mathrm{mmol})$ in $\mathrm{H}_{2} \mathrm{O}(18 \mathrm{~mL})$ it was added 2,5-dimethoxydihydrofuran (6) $(3.000 \mathrm{~g}, 23.051 \mathrm{mmol})$. The mixture was left under magnetic stirring at room temperature for $1 \mathrm{~h}$. It was added solid $\mathrm{NaHCO}_{3}$ until $\mathrm{pH} 7$ and then it was vigorously extracted with EtOAc $(2 \times 200 \mathrm{~mL})$. The organic layers were dried with $\mathrm{Na}_{2} \mathrm{SO}_{4}$, filtered, concentrated in vacuum and crude product was then purified by column chromatography eluted with EtOAc.

\section{3-Pyrrolin-2-one (9)}

$(0.681 \mathrm{~g}, 35 \%)$ as a brownish oil. $\mathrm{R}_{\mathrm{f}} 0.10$ (EtOAc). IR (film) $v_{\max } / \mathrm{cm}^{-1}: 3286,2920,1681,1448,1370,1244$, 1054, 807, 691. ${ }^{1} \mathrm{H}$ NMR $\left(200 \mathrm{MHz}, \mathrm{CDCl}_{3}\right): \delta 8.00$ (bs, $1 \mathrm{H}), 7.18(\mathrm{~m}, 1 \mathrm{H}), 6.17(\mathrm{~m}, 1 \mathrm{H}), 4.08(\mathrm{~m}, 2 \mathrm{H}) .{ }^{13} \mathrm{C} \mathrm{NMR}$ $\left(50 \mathrm{MHz}, \mathrm{CD}_{3} \mathrm{OD}\right): \delta 177.17(\mathrm{C}=\mathrm{O}), 148.93(\mathrm{CH})$, $127.55(\mathrm{CH}), 50.33\left(\mathrm{CH}_{2}\right) . \mathrm{m} / \mathrm{z}(\%): 83\left(\mathrm{M}^{+}, 72 \%\right), 55$ (100). Literature: ${ }^{14,15}$ IR (film) $v_{\max } / \mathrm{cm}^{-1}: 3250,1680$. ${ }^{1} \mathrm{H}$ NMR (100 MHz, acetone-d6): $\delta 8.00$ (bs, 1H), 7.30 $(\mathrm{m}, 1 \mathrm{H}), 6.10(\mathrm{~m}, 1 \mathrm{H}), 4.07(\mathrm{~m}, 2 \mathrm{H}) .{ }^{13} \mathrm{C}$ NMR: $\delta 175.5$ $(\mathrm{C}=\mathrm{O}), 147.6(\mathrm{CH}), 127.6(\mathrm{CH}), 49.5\left(\mathrm{CH}_{2}\right)$.

\section{4-Pyrrolin-2-one (10)}

$(0.097 \mathrm{~g}, 5 \%)$ as a brownish oil. $\mathrm{R}_{\mathrm{f}} 0.25$ (EtOAc). IR (film) $v_{\max } / \mathrm{cm}^{-1}: 3352,3098,2927,1694,1448,1352$, 1276, 1241, 1196, 1129, 1021, 806, 736. ${ }^{1} \mathrm{H}$ NMR (200 $\left.\mathrm{MHz}, \mathrm{CDCl}_{3}\right): \delta 8.39$ (bs, $\left.1 \mathrm{H}\right), 6.45(\mathrm{~m}, 1 \mathrm{H}), 5.26(\mathrm{~m}$, 1H), $3.02(\mathrm{~m}, 2 \mathrm{H})$. Literature: ${ }^{14}{ }^{1} \mathrm{H}$ NMR $(100 \mathrm{MHz}$, acetone-d6): $\delta 8.00(\mathrm{bs}, 1 \mathrm{H}), 6.59(\mathrm{~m}, 1 \mathrm{H}), 5.19(\mathrm{~m}$, $1 \mathrm{H}), 2.91(\mathrm{~m}, 2 \mathrm{H})$.

\section{$N$-(tert-butoxycarbonyl)-3-pyrrolin-2-one (4)}

To a solution of 3-pyrrolin-2-one (9) (4.698 g, 56.541 $\mathrm{mmol})$ in THF $(70 \mathrm{~mL})$, under magnetic stirring and room temperature, it was added DMAP $(0.345 \mathrm{~g}, 2.827 \mathrm{mmol})$ dissolved in THF $(10 \mathrm{~mL})$ and then (Boc) $)_{2} \mathrm{O}(13.574 \mathrm{~g}$, $62.195 \mathrm{mmol})$. After 10 minutes, the mixture was concentrated in vacuum, diluted with EtOAc $(100 \mathrm{~mL})$ and then washed with saturated solution of $\mathrm{NH}_{4} \mathrm{Cl}(1 \times 100$ $\mathrm{mL})$ and $\mathrm{H}_{2} \mathrm{O}(1 \times 100 \mathrm{~mL})$. The organic layer was separated and the aqueous phases were extracted with EtOAc $(2 \times$ $100 \mathrm{~mL}$ ). The combined organic layers were dried with $\mathrm{Na}_{2} \mathrm{SO}_{4}$, filtered, concentrated in vacuum and crude product was then purified by column chromatography eluted with $30 \%$ EtOAc/hexane and crescent gradient of EtOAc (50 and $100 \%)$. (4.187 $\mathrm{g}, 40 \%)$ as a brown oil. $\mathrm{R}_{\mathrm{f}} 0.29(50 \%$ EtOAc/hexane). IR (film) $v_{\max } / \mathrm{cm}^{-1}: 3097,2979,1768$, 1691, 1455, 1360, 1301, 1256, 1163, 1102, 1050, 971, 844, 817, 782, 701. ${ }^{1} \mathrm{H}$ NMR $\left(200 \mathrm{MHz}, \mathrm{CDCl}_{3}\right): \delta 7.18(\mathrm{dt}, J$ 2.1 and $6.1 \mathrm{~Hz}, 1 \mathrm{H}), 6.16(\mathrm{dt}, J 2.0$ and $6.1 \mathrm{~Hz}, 1 \mathrm{H}), 4.35$ (t, $J 2.0 \mathrm{~Hz}, 2 \mathrm{H}), 1.56(\mathrm{~s}, 9 \mathrm{H}) .{ }^{13} \mathrm{C} \mathrm{NMR}\left(50 \mathrm{MHz}, \mathrm{CDCl}_{3}\right)$ : $\delta 168.89(\mathrm{C}=\mathrm{O}), 149.22(\mathrm{C}=\mathrm{O}), 145.02(\mathrm{CH}), 127.53(\mathrm{CH})$, $82.61(\mathrm{C}), 51.39\left(\mathrm{CH}_{2}\right), 27.79\left(3 \mathrm{CH}_{3}\right) . \mathrm{m} / z(\%): 183\left(\mathrm{M}^{+}\right.$, 1\%), 128 (65), 110 (48), 84 (20), 56 (100), 44 (68).

\section{$N$-(tert-butoxycarbonyl)-4-nitromethylene-2-pyrrolidinone (11)}

To a mixture of $\mathrm{MeNO}_{2}(0.44 \mathrm{~mL}, 8.185 \mathrm{mmol}), \mathrm{DBU}$ $(0.24 \mathrm{~mL}, 1.637 \mathrm{mmol})$ and $\mathrm{MeCN}(2.0 \mathrm{~mL})$, under magnetic stirring and room temperature, it was added $\mathrm{N}$ (tert-butoxycarbonyl)-3-pyrrolin-2-one (4) (0.300 g, 1.637 mmol) dissolved in $\mathrm{MeCN}(3.5 \mathrm{~mL})$. After $1 \mathrm{~h}$, the mixture was diluted with EtOAc $(50 \mathrm{~mL})$ and washed with saturated solution of $\mathrm{NH}_{4} \mathrm{Cl}(1 \times 30 \mathrm{~mL})$. The organic layer was separated and the aqueous phase was extracted with EtOAc $(2 \times 50 \mathrm{~mL})$. The combined organic layers were dried with $\mathrm{Na}_{2} \mathrm{SO}_{4}$, filtered, concentrated in vacuum and crude product was then purified by column chromatography eluted with $50 \% \mathrm{EtOAc} /$ hexane. $(0.160 \mathrm{~g}, 40 \%)$ as a yellowish crystalline residue. $\mathrm{R}_{\mathrm{f}} 0.47$ (60\% EtOAc/hexane). IR (KBr) $v_{\max } / \mathrm{cm}^{-1}: 2983,1779,1552,1373,1311,1258,1156,1023$, 946, 778, 749, 643. ' $\mathrm{H}$ NMR (200 MHz, $\left.\mathrm{CDCl}_{3}\right): \delta 4.48(\mathrm{~d}$, 
$J 7.4 \mathrm{~Hz}, 2 \mathrm{H}), 4.03$ (dd, $J 7.8$ and $11.4 \mathrm{~Hz}, 1 \mathrm{H}), 3.55$ (dd, $J$ 6.4 and $11.4 \mathrm{~Hz}, 1 \mathrm{H}), 3.13(\mathrm{~m}, 1 \mathrm{H}), 2.80$ (dd, $J 8.7$ and $17.5 \mathrm{~Hz}, 1 \mathrm{H}$ ), 2.36 (dd, J 7.6 and $17.5 \mathrm{~Hz}, 1 \mathrm{H}$ ), 1.53 (s, 9H). ${ }^{13} \mathrm{C} \mathrm{NMR}\left(50 \mathrm{MHz}, \mathrm{CDCl}_{3}\right): \delta 171.13(\mathrm{C}=\mathrm{O}), 149.14$ $(\mathrm{C}=\mathrm{O}), 83.20(\mathrm{C}), 76.71\left(\mathrm{CH}_{2}\right), 48.80\left(\mathrm{CH}_{2}\right), 35.82\left(\mathrm{CH}_{2}\right)$, $28.49(\mathrm{CH}), 27.63\left(3 \mathrm{CH}_{3}\right)$.

\section{Supplementary Information}

NMR spectra for the compounds $\mathbf{4 ,} \mathbf{8} \mathbf{a}-\mathbf{b}$ and $\mathbf{9 - 1 1}$ are available free of charge as PDF file at http://jbcs.sbq.org.br.

\section{Acknowledgments}

J. C. F. A. thanks Department of Organic Chemistry (IQUFF) for request a fellowship to develop this work, FAPERJ (E-26/150.293/2001) for the concession of the fellowship, NPPN-UFRJ for space (LQB) to perform the experimental work and for NMR spectra, and to analytical centrals (Dequim-UFRRJ, IQ-UFRJ, IQ-UFF) for infrared spectra. J. C. F. A. also thanks the Laboratory of Glycobiology (IBCCF-UFRJ) for the measurements of specific optical rotation and to Dequim-UFRRJ for mass spectra.

\section{References}

1. Merino, P.; Castillo, E.; Franco, S.; Merchan, F. L.; Tejero, T.; Tetrahedron: Asymmetry 1998, 9, 1759 and references cited therein.

2. Hussaini, S. R.; Moloney, M. G.; Org. Biomol. Chem. 2006, 4, 2600; Moloney, M. G.; Panchal, T.; Pike, R.; Org. Biomol. Chem. 2006, 4, 3894.

3. Moloney, M. G.; Nat. Prod. Rep. 2002, 19, 597.

4. Qiao, L.; Zhao, L. Y.; Rong, S. B.; Wu, X. W.; Wang, S.; Fujii, T.; Kazanietz, M. G.; Rauser, L.; Savage, J.; Roth, B. L.; Flippen-Anderson, J.; Kozikowski, A. P.; Bioorg. Med. Chem. Lett. 2001, 11, 955.
5. Wu, B.; Kuhen, K.; Nguyen, T. N.; Ellis, D.; Anaclerio, B.; He, X.; Yang, K.; Karanewsky, D.; Yin, H.; Wolff, K.; Bieza, K.; Caldwell, J.; He, Y.; Bioorg. Med. Chem. Lett. 2006, 16, 3430.

6. Smith-III, A. B.; Charnley, A. K.; Harada, H.; Beiger, J. J.; Cantin, L. D.; Kenesky, C. S.; Hirschmann, R.; Munshi, S.; Olsen, D. B.; Stahlhut, M. W.; Schleif, W. A. ; Kuo, L. C.; Bioorg. Med. Chem. Lett. 2006, 16, 859.

7. Baussanne, I.; Chiaroni, A.; Husson, P. H.; Riche, C.; Royer, J.; Tetrahedron Lett. 1994, 35, 3931.

8. Herdeis, C.; Hubmann, H. P.; Tetrahedron: Asymmetry 1992, 3, 1213; Diaz, A.; Siro, J. G.; García-Navío, J. L.; Vaquero, J. J.; Alvarez-Builla, J.; Synthesis 1997, 559.

9. Recent syntheses of polysubstituted 3- and 4-pyrrolin-2-ones were described by: Murai, M.; Kawai, S.; Miki, K.; Ohe, K.; J. Organomet. Chem. 2007, 692, 579; Demir, A. S.; Emrullahoglu, M.; Ardahan, G.; Tetrahedron 2007, 63, 461.

10. Casiraghi, G.; Rassu, G.; Spanu, P.; Pinna, L.; J. Org. Chem. 1992, 57, 3760.

11. Costa, J. S.; Dias, A. G.; Anholeto, A. L.; Monteiro, M. D.; Patrocínio, V. L.; Costa, P. R. R.; J. Org. Chem. 1997, 62, 4002.

12. Peçanha, E. P.; Fraga, C. A. M.; Barreiro, E. J.; Braga, M. F. M.; Pereira, E. F. R.; Albuquerque, E. X.; J. Braz. Chem. Soc. 2001, 12, 408.

13. Perrin, D. D.; Armarego, W. L. F.; Perrin, D. R.; Purification of Laboratory Chemicals, $2^{\text {nd }}$ ed.; Pergamon Press Ltd: New York, 1980.

14. Bocchi, V.; Chierici, L.; Gardini, G. P.; Mondelli, R.; Tetrahedron 1970, 26, 4073.

15. Pretsch, E.; Seibl, J.; Simon, W.; Clerc, T.; Tables of Spectral Data for Structure Determination of Organic Compounds, $2^{\text {nd }}$ ed.; Fresenius,W.; Huber, J. F. K.; Pungor, E.; Rechnitz, G. A.; Simon, W.; West, T. S., Ed.; Springer-Verlag: Berlin, 1989, p. C197.

Received: January 17, 2007 Web Release Date: July 18, 2007 
Preliminary Studies towards the Preparation of Reactive 3-Pyrrolin-2-ones in Conjugate Addition Reactions for the Syntheses of Potentially Bioactive 2-Pyrrolidinones and Pyrrolidines

José C. F. Alves*
Instituto de Química, Universidade Federal Fluminense, Outeiro de S. João Batista s/n, Centro,
24210-150 Niterói-RJ, Brazil
Núcleo de Pesquisas de Produtos Naturais, Universidade Federal do Rio de Janeiro,
CCS, Bloco H, 21941-590 Rio de Janeiro-RJ, Brazil

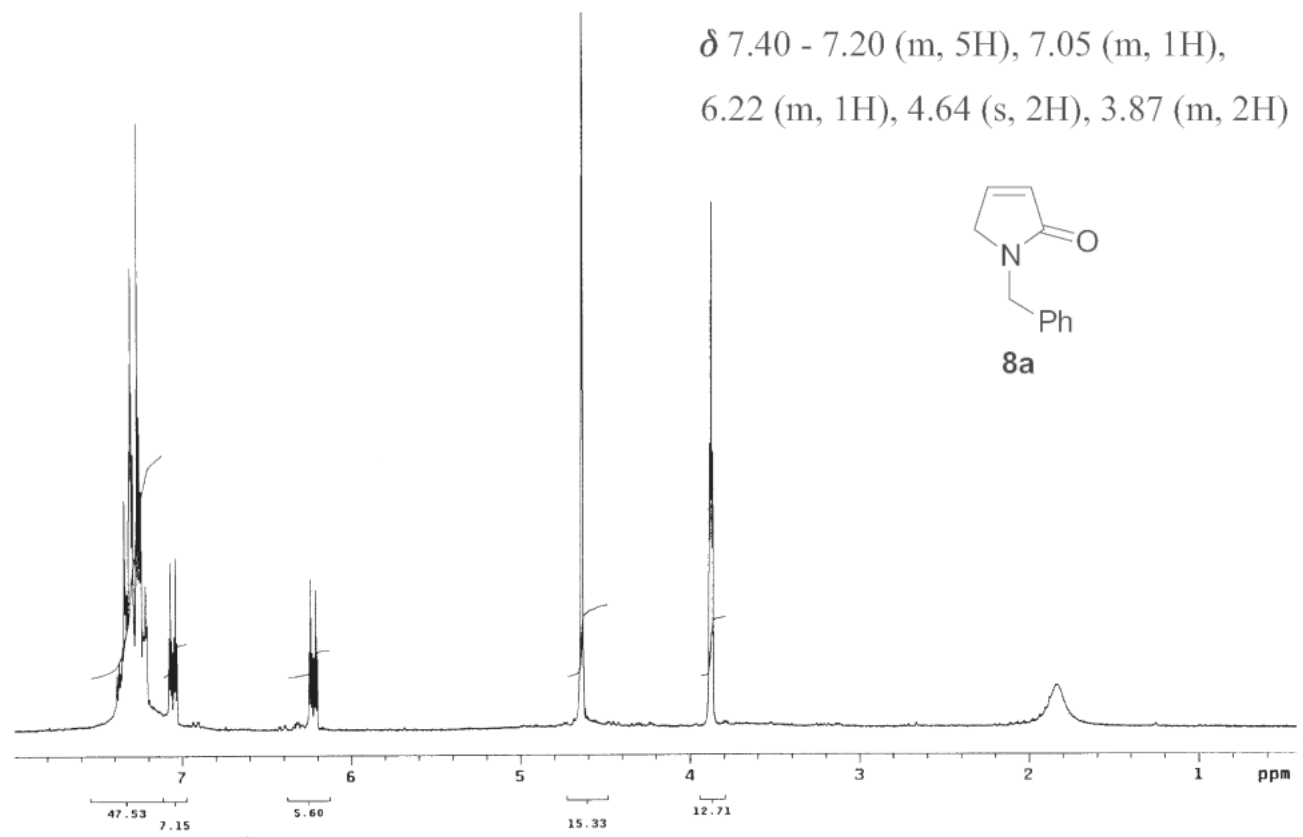

Figure S1. ${ }^{1} \mathrm{H}$ NMR spectrum of compound $8 \mathbf{8}\left(200 \mathrm{MHz}, \mathrm{CDCl}_{3}\right)$.

*e-mail: alvesjcf@yahoo.com.br 


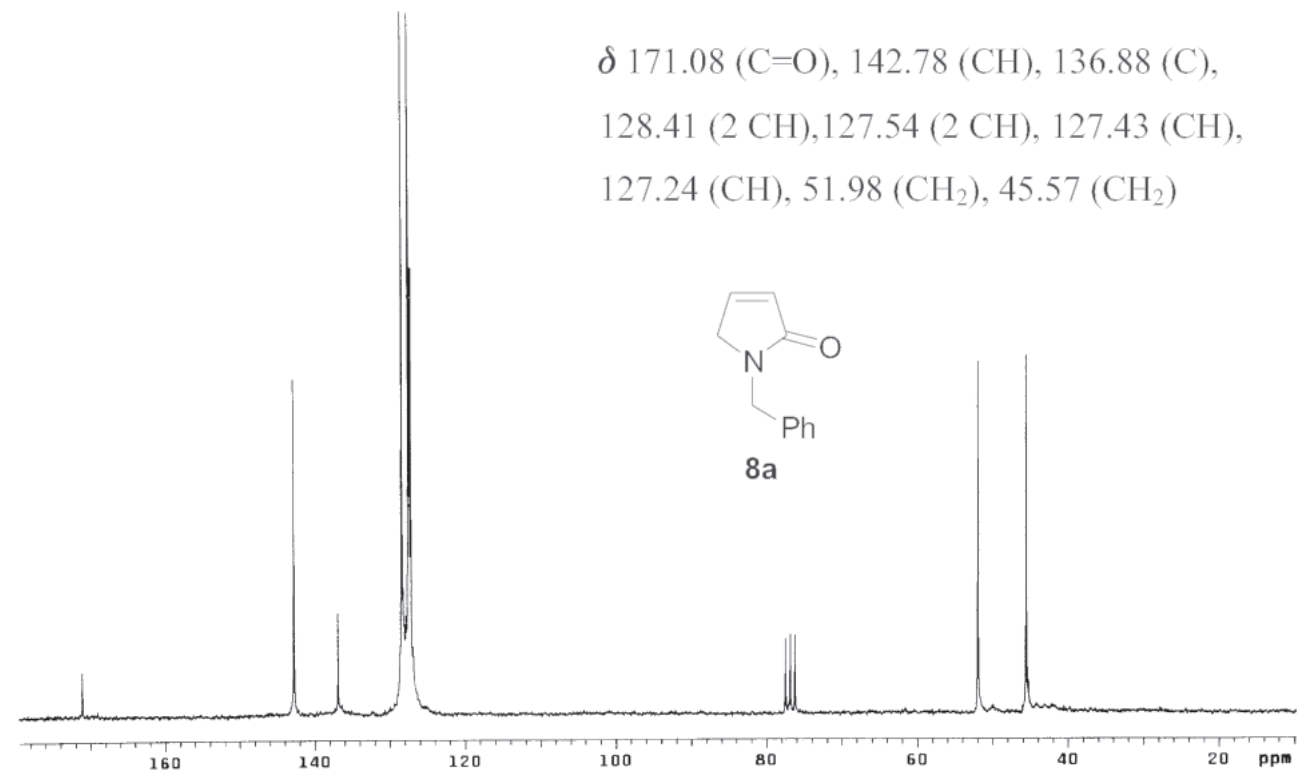

Figure S2. ${ }^{13} \mathrm{C}$ NMR spectrum of compound $8 \mathbf{8}\left(50 \mathrm{MHz}, \mathrm{CDCl}_{3}\right)$.

$\delta 7.40-7.21(\mathrm{~m}, 5 \mathrm{H}), 7.03(\mathrm{~m}, 1 \mathrm{H}), 6.18(\mathrm{~m}, 1 \mathrm{H})$,

$5.58(\mathrm{q}, J 7.1 \mathrm{~Hz}, 1 \mathrm{H}), 3.94$ (dt, $J 1.8$ and $20.4 \mathrm{~Hz}, 1 \mathrm{H})$,

$3.62(\mathrm{dt}, J 1.8$ and $20.4 \mathrm{~Hz}, 1 \mathrm{H}), 1.61(\mathrm{~d}, J 7.1 \mathrm{~Hz}, 3 \mathrm{H})$

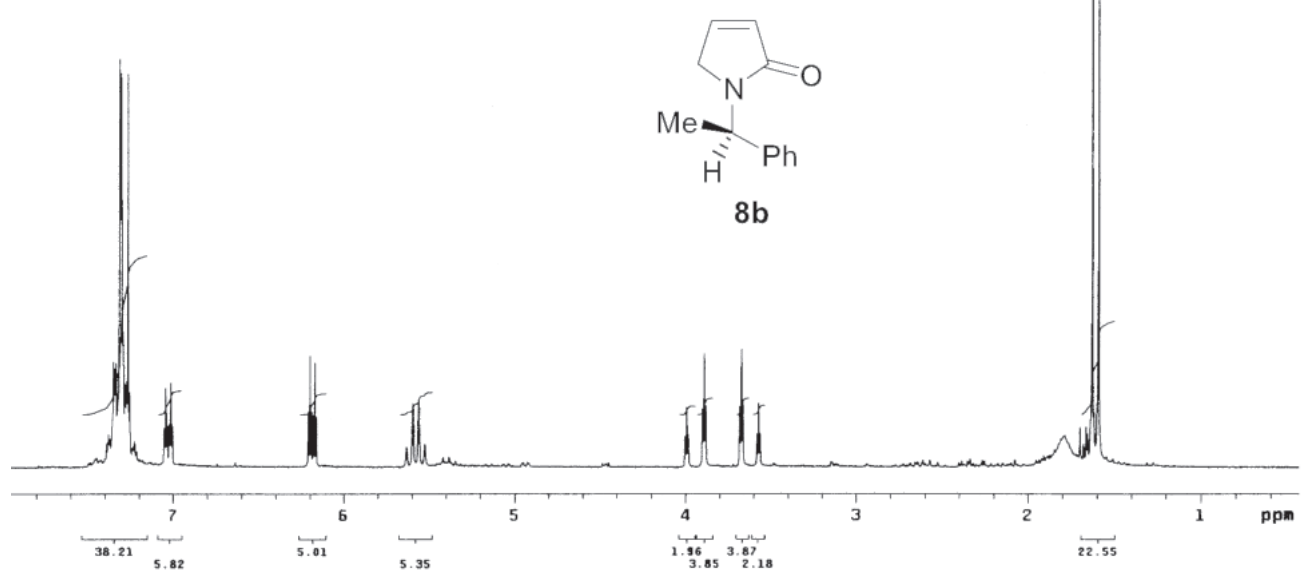

Figure S3. ${ }^{1} \mathrm{H}$ NMR spectrum of compound $\mathbf{8 b}\left(200 \mathrm{MHz}, \mathrm{CDCl}_{3}\right)$. 


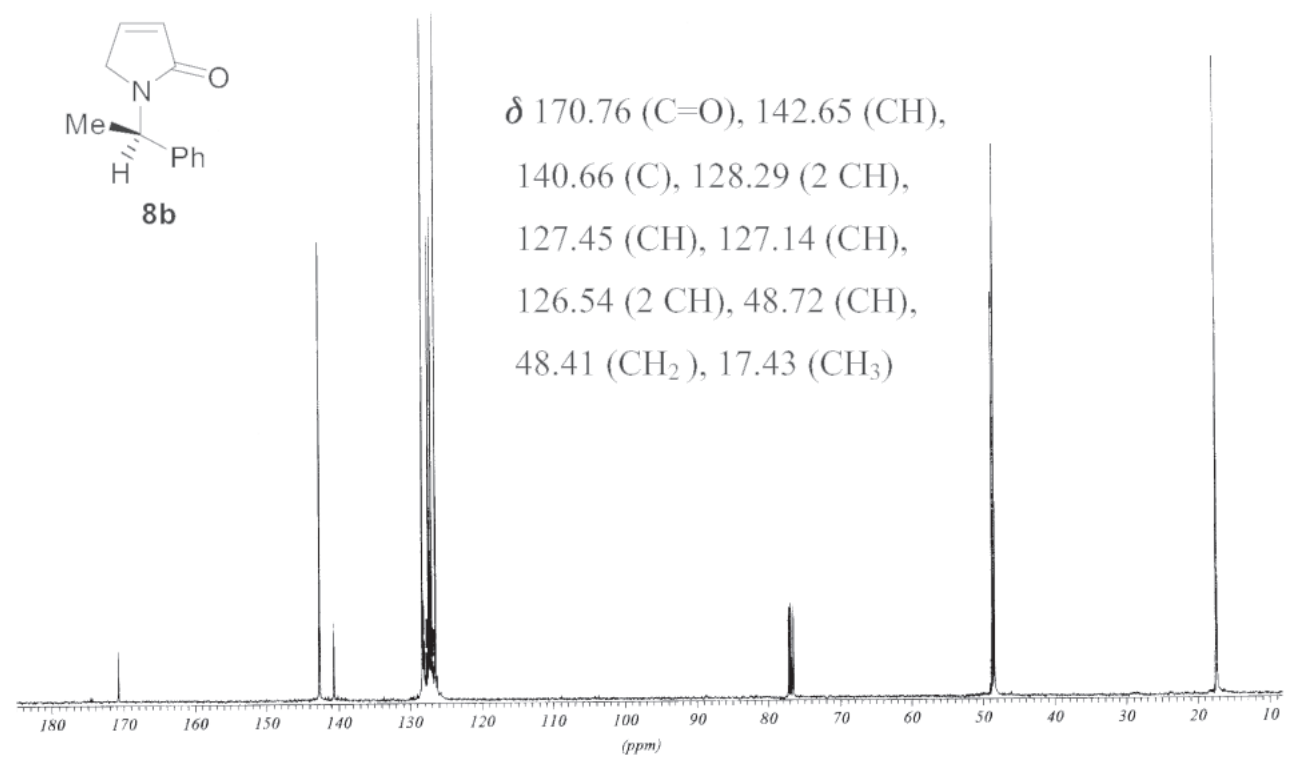

Figure S4. ${ }^{13} \mathrm{C}$ NMR spectrum of compound $\mathbf{8 b}\left(100 \mathrm{MHz}, \mathrm{CDCl}_{3}\right)$.

$\delta 8.00(\mathrm{bs}, 1 \mathrm{H}), 7.18(\mathrm{~m}, 1 \mathrm{H})$,

$6.17(\mathrm{~m}, 1 \mathrm{H}), 4.08(\mathrm{~m}, 2 \mathrm{H})$

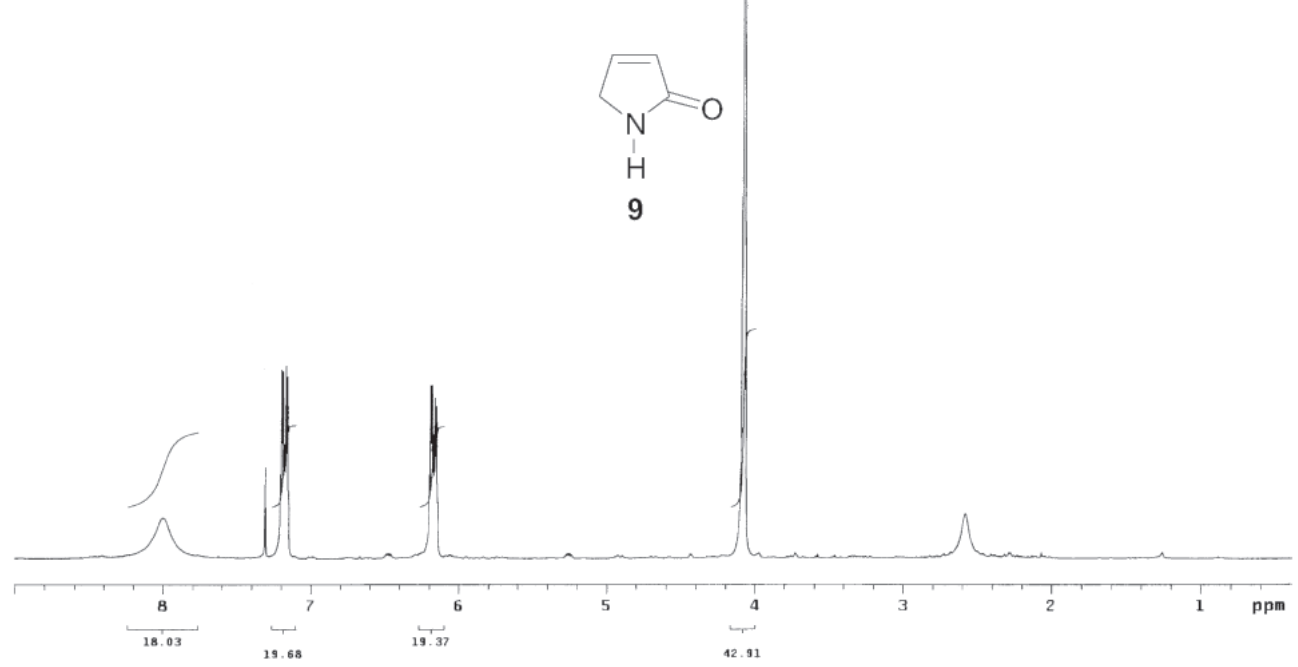

Figure S5. ${ }^{1} \mathrm{H}$ NMR spectrum of compound $9\left(200 \mathrm{MHz}, \mathrm{CDCl}_{3}\right)$. 


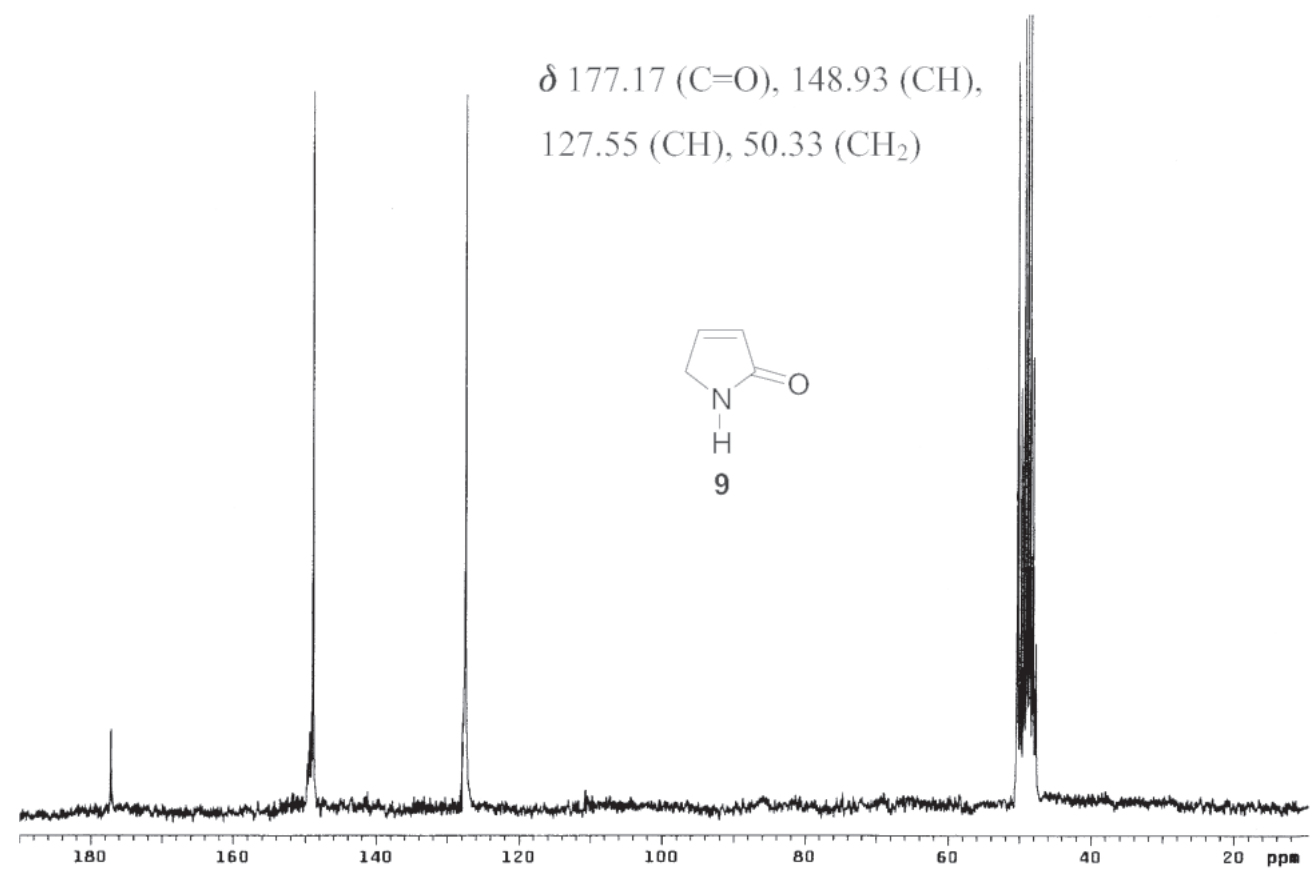

Figure S6. ${ }^{13} \mathrm{C}$ NMR spectrum of compound $9\left(50 \mathrm{MHz}, \mathrm{CD}_{3} \mathrm{OD}\right)$.

$\delta 8.39(\mathrm{bs}, 1 \mathrm{H}), 6.45(\mathrm{~m}, 1 \mathrm{H}), 5.26(\mathrm{~m}, 1 \mathrm{H}), 3.02(\mathrm{~m}, 2 \mathrm{H})$

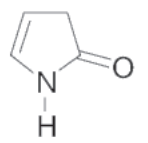

10

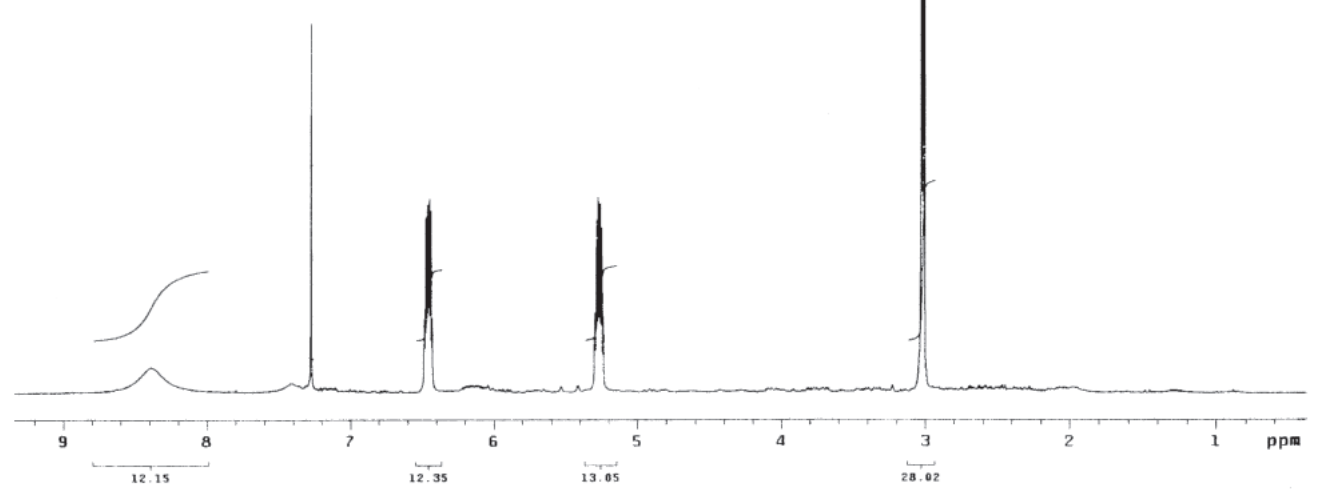

Figure S7. ${ }^{1} \mathrm{H}$ NMR spectrum of compound $10\left(200 \mathrm{MHz}, \mathrm{CDCl}_{3}\right)$. 
$\delta 7.18(\mathrm{dt}, J 2.1$ and $6.1 \mathrm{~Hz}, 1 \mathrm{H})$,

$6.16(\mathrm{dt}, J 2.0$ and $6.1 \mathrm{~Hz}, 1 \mathrm{H})$,

$4.35(\mathrm{t}, J 2.0 \mathrm{~Hz}, 2 \mathrm{H}), 1.56(\mathrm{~s}, 9 \mathrm{H})$
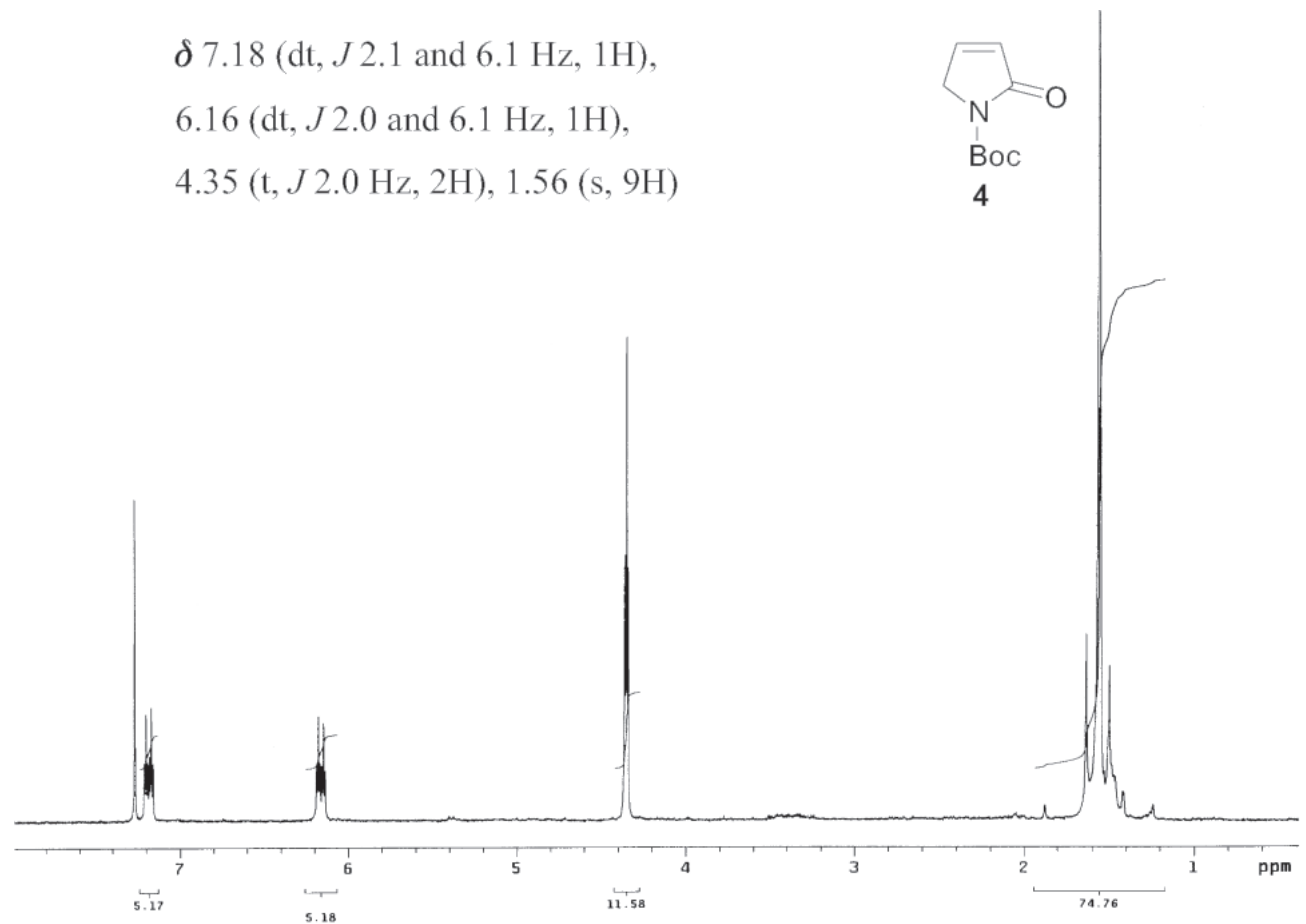

Figure S8. ${ }^{1} \mathrm{H}$ NMR spectrum of compound $4\left(200 \mathrm{MHz}, \mathrm{CDCl}_{3}\right)$.

$\delta 168.89(\mathrm{C}=\mathrm{O}), 149.22(\mathrm{C}=\mathrm{O})$,

$145.02(\mathrm{CH}), 127.53(\mathrm{CH})$,

$82.61(\mathrm{C}), 51.39\left(\mathrm{CH}_{2}\right), 27.79\left(3 \mathrm{CH}_{3}\right)$
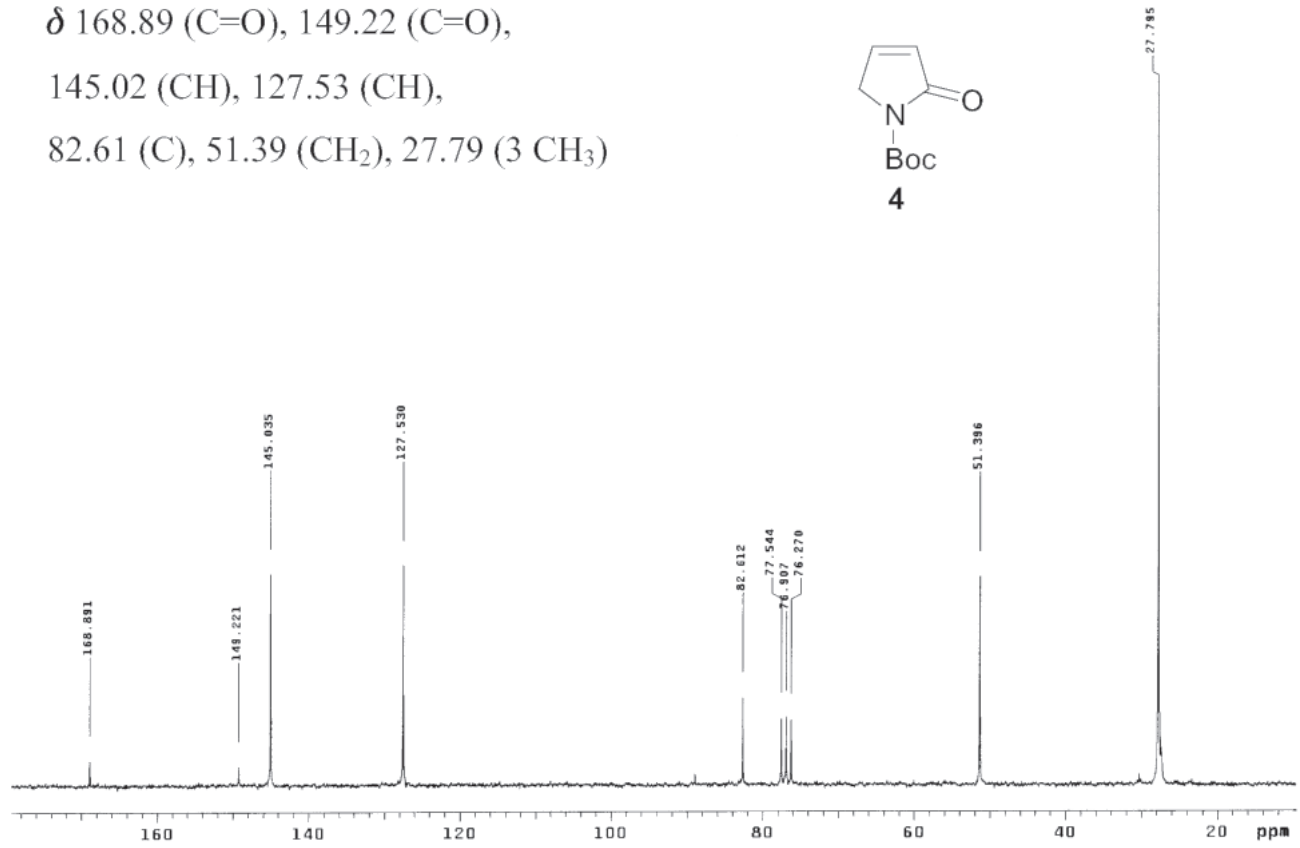

Figure S9. ${ }^{13} \mathrm{C}$ NMR spectrum of compound $4\left(50 \mathrm{MHz}, \mathrm{CDCl}_{3}\right)$. 
$\delta 4.48$ (d, $J 7.4 \mathrm{~Hz}, 2 \mathrm{H}), 4.03$ (dd, $J 7.8$ and $11.4 \mathrm{~Hz}, 1 \mathrm{H})$,

$3.55(\mathrm{dd}, J 6.4$ and $11.4 \mathrm{~Hz}, 1 \mathrm{H}), 3.13(\mathrm{~m}, 1 \mathrm{H})$,

$2.80(\mathrm{dd}, J 8.7$ and $17.5 \mathrm{~Hz}, 1 \mathrm{H})$,

$2.36(\mathrm{dd}, J 7.6$ and $17.5 \mathrm{~Hz}, 1 \mathrm{H}), 1.53(\mathrm{~s}, 9 \mathrm{H})$

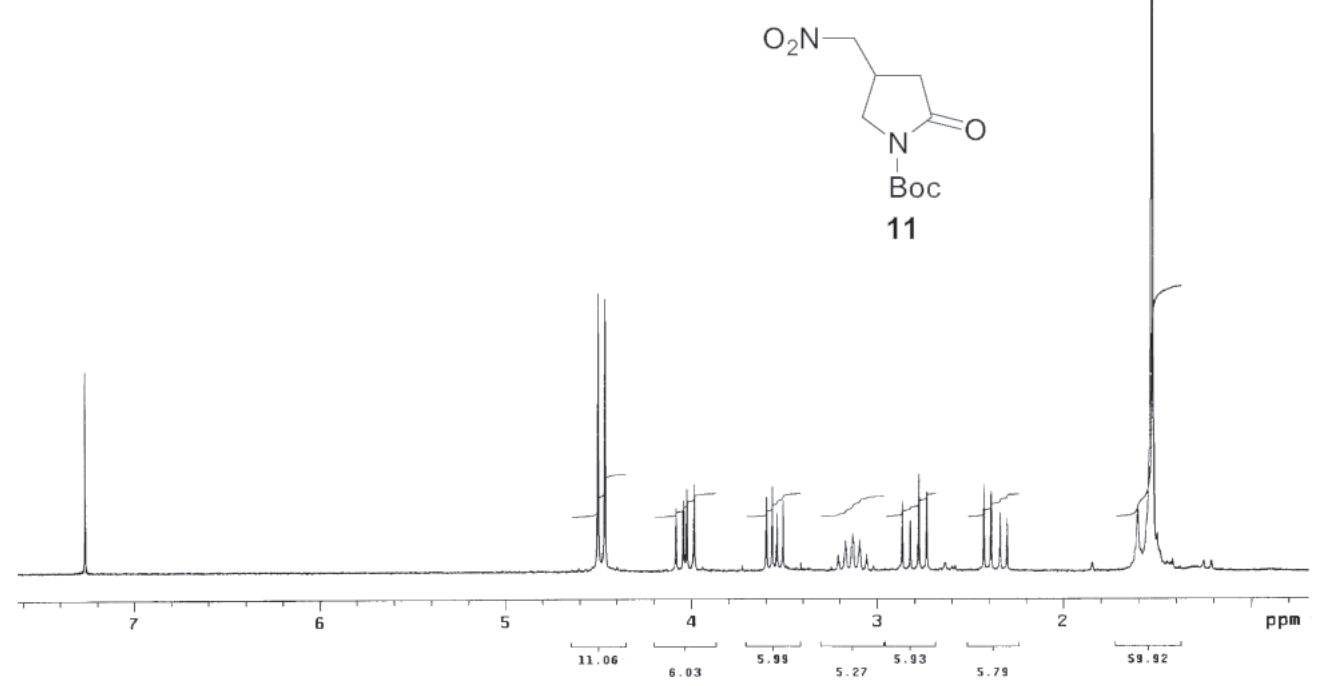

Figure S10. ${ }^{1} \mathrm{H}$ NMR spectrum of compound $11\left(200 \mathrm{MHz}, \mathrm{CDCl}_{3}\right)$.

$\delta 171.13(\mathrm{C}=\mathrm{O}), 149.14(\mathrm{C}=\mathrm{O})$,

$83.20(\mathrm{C}), 76.71\left(\mathrm{CH}_{2}\right), 48.80\left(\mathrm{CH}_{2}\right)$,

$35.82\left(\mathrm{CH}_{2}\right), 28.49(\mathrm{CH}), 27.63\left(3 \mathrm{CH}_{3}\right)$

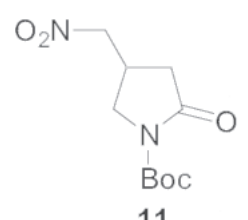

11

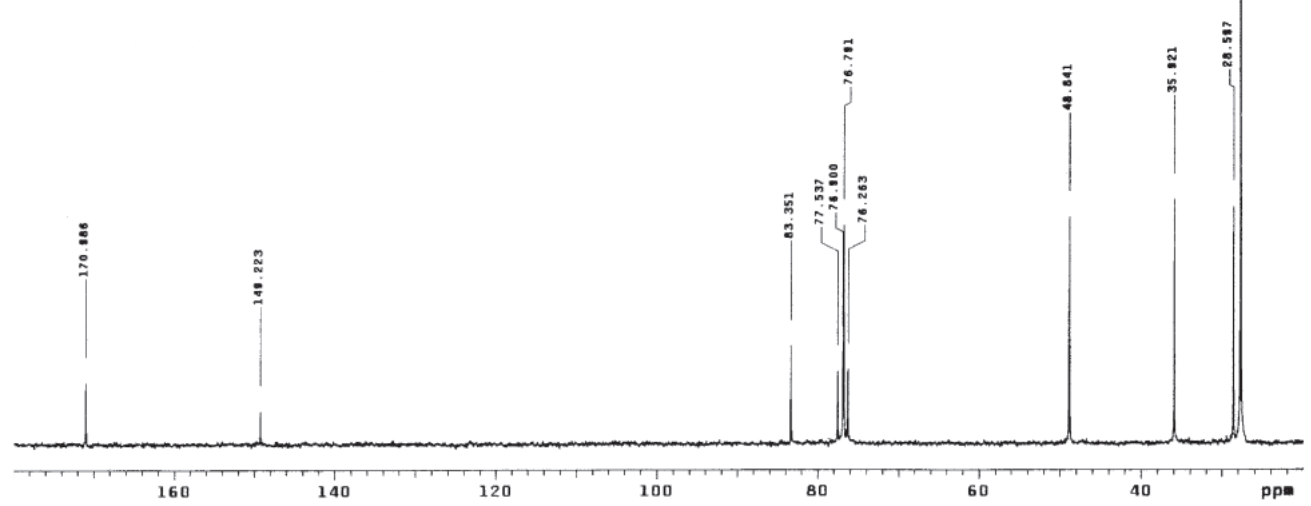

Figure S11. ${ }^{13} \mathrm{C}$ NMR spectrum of compound $11\left(50 \mathrm{MHz}, \mathrm{CDCl}_{3}\right)$. 